\title{
Os Polioxometalatos. Do Anião de Keggin às Nanocápsulas
}

\author{
JOSÉ GAMELAS*, ANA CAVALEIRO, ISABEL SANTOS, MARIA SALETE BALULA
}

\section{Resumo}

Uma grande parte da investigação que se faz em Química tem como objectivo a síntese e estudo de compostos químicos (ou materiais) que possuam certas propriedades desejadas: um material com determinadas propriedades condutoras da corrente eléctrica, uma substância que catalise um certo tipo de reacções químicas, um composto inibidor da acção de vírus (ou bactérias) prejudiciais à saúde, etc. A obtenção de substâncias capazes de desempenhar múltiplas funções é, neste contexto, um de-

safio máximo que se propõe ao investigador. Neste artigo, pretende-se apresentar ao leitor um tipo particular de espécies químicas, os polioxometalatos. Estes aniões apresentam um conjunto de propriedades estruturais e electrónicas que os tornam úteis em áreas tão diversas como a catálise, medicina, ciência dos materiais ou química analítica. Considerações sobre a síntese, propriedades e aplicações mais relevantes dos polioxometalatos serão aqui contempladas.

\section{Introdução. Perspectiva histórica}

Os polioxometalatos (de fórmula geral $\left[\mathrm{M}_{\mathrm{x}} \mathrm{O}_{\mathrm{y}}\right]^{\mathrm{m}-\mathrm{ou}}\left[\mathrm{X}_{\mathrm{z}} \mathrm{M}_{\mathrm{x}} \mathrm{O}_{\mathrm{y}}\right]^{\mathrm{n}-}, \mathrm{z} \leq \mathrm{x}, \mathrm{M}=\mathrm{Mo}, \mathrm{W}$, $\mathrm{V}, \mathrm{Nb}, \mathrm{Ta}$ e $\mathrm{X}$ variável) são aniões que se podem estruturalmente visualizar como agregados obtidos a partir da condensação de poliedros metal-oxigénio, $\mathrm{MO}_{\mathrm{x}}$ : octaedros (o mais frequente), bipirâmides pentagonais ou pirâmides quadrangulares, em que o metal M está deslocado da posição central do poliedro em direcção aos oxigénios não partilhados (devido à formação de ligações $\pi \mathrm{M}-0$ ). [1-6] Nas figuras 1 e 2 ilustram-se as estruturas de alguns tipos de polioxometalatos conhecidos.

0 primeiro polioxometalato que se conhece foi preparado em 1826 quando Berzelius obteve um precipitado amarelo da reacção entre molibdato de amónio e ácido fosfórico, o 12-molibdofosfato de amónio. A partir desta descoberta, diversos polioxomolibdatos ( $\mathrm{M}=\mathrm{Mo}$ ) e polioxotungstatos $(M=W)$ foram referi- dos, sendo valorizada a sua aplicação em química analítica, para determinações gravimétricas tais como as de P ou Si. Só nas primeiras décadas do século $\mathrm{XX}$ serão propostas as primeiras teorias para explicar a estrutura deste tipo de compostos (teoria de coordenação de Werner, teoria de Miolati-Rosenheim, proposta estrutural de Pauling). A mais "correcta" foi a de Linus Pauling, que em 1929, propôs estruturas baseadas em octaedros $\mathrm{MO}_{6}$ ligados entre si por partilha de vértices de oxigénio. $[2,4,6]$ A determinação estrutural do heteropoliácido $\mathrm{H}_{3}\left[\mathrm{PW}_{12} \mathrm{O}_{40}\right] .6 \mathrm{H}_{2} \mathrm{O}$ (actualmente formulado como $\left[\mathrm{H}_{5} \mathrm{O}_{2}\right]_{3}\left[\mathrm{PW}_{12} \mathrm{O}_{40}\right]$ ) feita por Keggin em 1933 foi o primeiro grande passo para compreender a estrutura deste tipo de compostos. Com base no correspondente difractograma de raios $X$ de pós, mostrou que 0 anião apresenta uma estrutura compacta baseada num arranjo de octaedros $\mathrm{WO}_{6}$ ligados entre si por partilha de arestas e vértices de oxigénios (figura 1c). [9]
A síntese de novos polioxometalatos teve grande desenvolvimento durante todo o século XX. No entanto, dado as grandes dimensões e complexidade estrutural da maior parte destes aniões a sua caracterização sempre se apresentou de grande dificuldade até há relativamente pouco tempo. Nos anos 60, os estudos de caracterização limitavam-se, em geral, a análises químicas (grande empenho na exactidão/precisão das determinações analíticas), determinação de massas molares, estudos polarográficos, caracterização por espectroscopia vibracional e electrónica. As determinações cristalográficas eram escassas (até 1971, segundo um artigo de revisão, registavam-se cerca de 25 e algumas destas com pouca qualidade) e certas técnicas não tinham, ainda, sensibilidade suficiente para o estudo deste tipo de compostos. $[1,2,4,6]$ Hoje, com o desenvolvimento das técnicas de Ressonância Magnética Nuclear (183W, 95Mo,

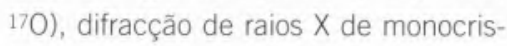
tal e espectrometria de massa, entre ou-

\footnotetext{
* Departamento de Química, Universidade de Aveiro, 3810-193 Aveiro, Portugal

*E-mail: jgamelas@dq.ua.pt
} 
figura 1 Representação poliédrica da estrutura de vários polioxometalatos. a) anião de Lindquist, $\left[\mathrm{W}_{6} \mathrm{O}_{19}\right]^{2-;}$ b) anião de Andersen, $\left[\mathrm{TeMo}_{6} \mathrm{O}_{24}\right]^{6-; c)}$ anião de Keggin, $\left[\mathrm{PW}_{12} \mathrm{O}_{40}\right]^{3}$; d) anião de Dawson, $\left.\left[\mathrm{P}_{2} \mathrm{~W}_{18} \mathrm{O}_{68}\right]^{6} ; e\right)$

$\left[\mathrm{Co}_{4}\left(\mathrm{H}_{2} \mathrm{O}\right)_{2}\left(\mathrm{PW}_{9} \mathrm{O}_{34}\right)_{2}\right]^{10}$. [2] Observe-se a presença de octaedros $\mathrm{MO}_{6}$ em todos os aniões.
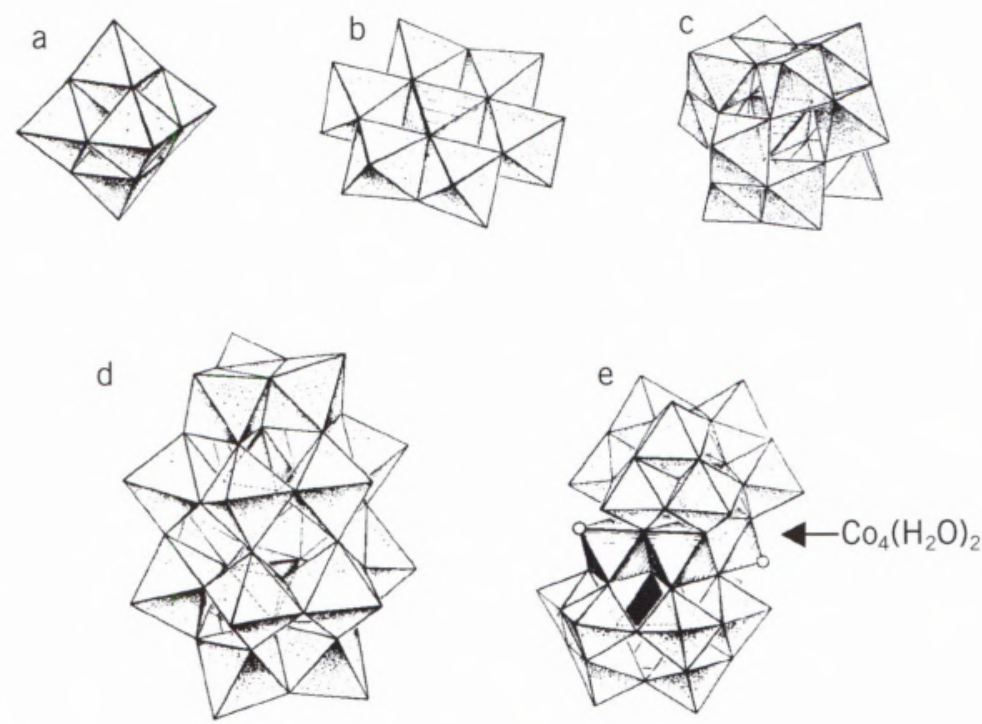

figura 2 Representação poliédrica da estrutura de polioxometalatos de grandes dimensões e dos respectivos fragmentos constituintes a) $\left[\mathrm{Mo}_{176} \mathrm{O}_{528}\left(\mathrm{H}_{2} \mathrm{O}\right){ }_{80} \mathrm{H}_{n}\right]^{(32 \cdot n)} \cdot=\left\{\mathrm{Mo}_{176}\right\} ;[7]$ b) $\left[\mathrm{Mo}_{132} \mathrm{O}_{372}\left(\mathrm{CH}_{3} \mathrm{COO}\right)_{30}\left(\mathrm{H}_{2} \mathrm{O}\right)_{72}\right]^{42}=\left\{\mathrm{Mo}_{132}\right\}$. [8] Na estrutura a com forma semelhante a um anel, fragmentos $\left[\mathrm{Mo}_{8}\right]$ estão ligados entre si por intermédio dos fragmentos $\left\{\mathrm{Mo}_{2}\right\}$ e $\left\{\mathrm{Mo}_{2}\right\}$ (estes localizados no plano equatorial do anel) $\mathrm{Na}$ estrutura $\underline{b}$ com forma aproximadamente esférica, os vários fragmentos $\{$ Mo6 $\}$ ligam-se entre si por intermédio de pontes $\left\{\mathrm{Mo}_{2} \mathrm{O}_{4}\left(\mathrm{CH}_{3} \mathrm{COO}\right)\right\}$.

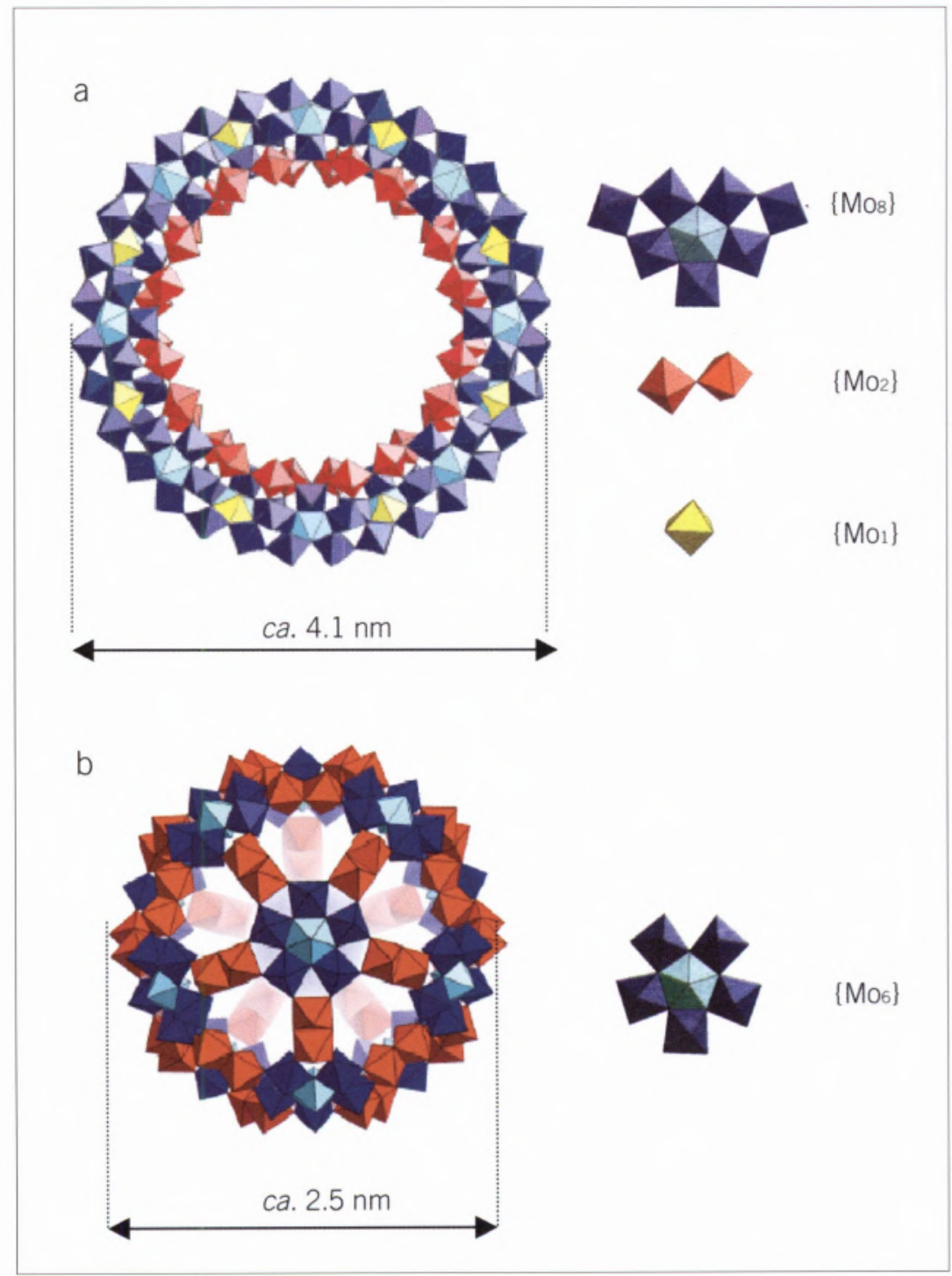




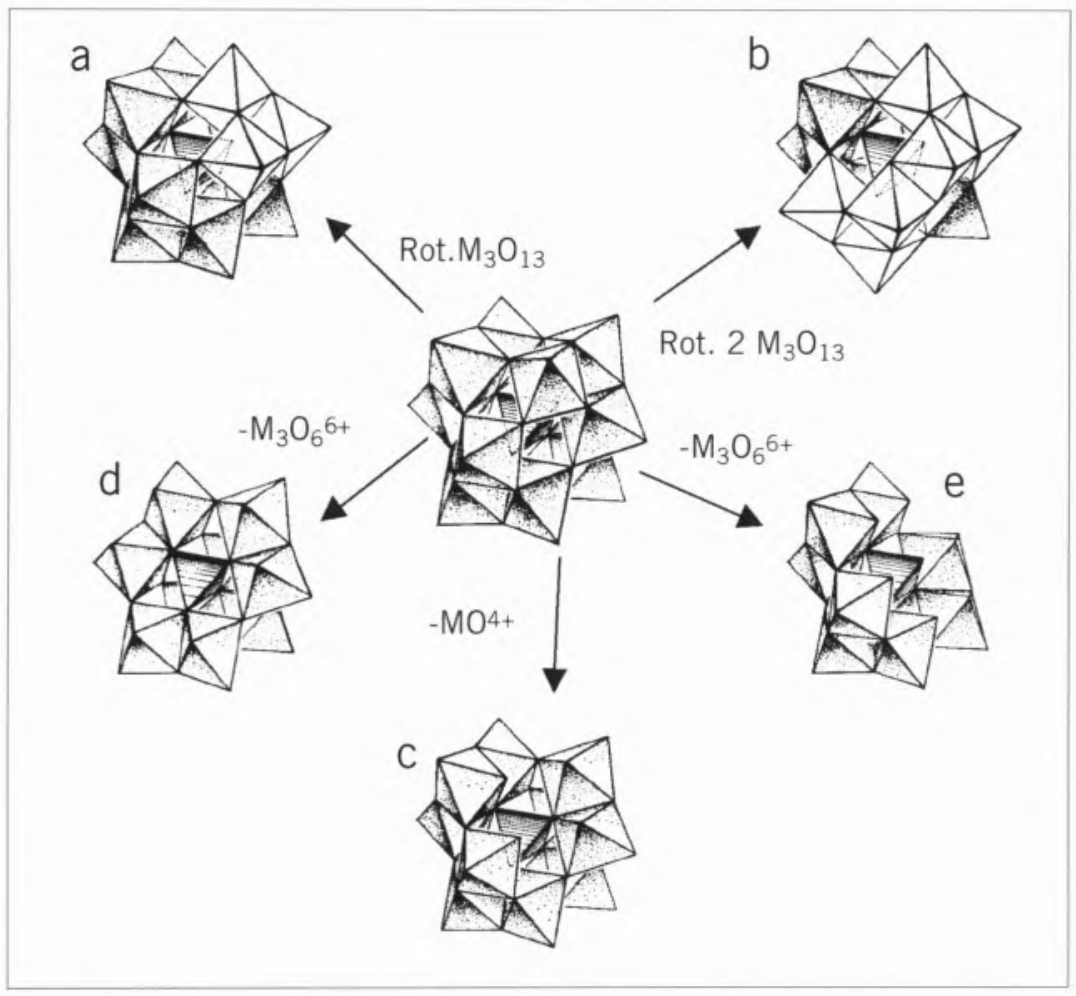

tras, já é possivel obter uma informação estrutural mais precisa sobre este família complexa de compostos.

0 domínio de investigação dos polioxometalatos é bastante ramificado, incluindo a síntese de novos materiais e estudo das suas propriedades físicas (propriedades ópticas incluindo óptica não linear, propriedades magnéticas), utilização em catálise ácida ou oxidativa, avaliação do potencial em medicina (ex: agentes anti-virais) ou ainda a concepção de novas arquitecturas moleculares com dimensões nanométricas. [5,10,11]

\section{Características típicas}

Nos polioxometalatos podem-se considerar duas familias representativas, os isopolianiões $\left(\left[\mathrm{M}_{\mathrm{x}} \mathrm{O}_{\mathrm{y}}\right]^{\mathrm{m}-}\right)$ e os heteropolianiões $\left(\left[\mathrm{X}_{z} \mathrm{M}_{\mathrm{x}} \mathrm{O}_{\mathrm{y}}\right]^{\mathrm{n}-\mathrm{-}}\right)$. Os isopolianiões são aniões constituídos por dois tipos de elementos: o oxigénio e um elemento metálico $M=M o, W$ (ou os menos frequentes $\mathrm{V}, \mathrm{Nb}$ e $\mathrm{Ta}$ ). Este segundo elemento combina carga e raio iónico apropriados com orbitais $\delta$ vazias para a ligação $\quad \pi \mathrm{M}-\mathrm{O}$. Nos heteropolianiōes já aparece incorporado um outro elemento, o heteroátomo $\mathrm{X}$, podendo haver um ou mais heteroátomos (conhecem-se mais de 70 heteroelementos diferentes). O heteroátomo primário é aquele que imprescindivel para a manutenção da estrutura básica do heteropolianião; este não pode ser removido ou substituido quimicamente, sem destruir o anião. O heteroátomo secundário pode por sua vez ser libertado do heteropolianião deixando uma sub-unidade polioxoaniónica estável. [2,4] Por exemplo, no heteropolianião $\alpha$ $\left[\mathrm{PW}_{11} \mathrm{Co}\left(\mathrm{H}_{2} \mathrm{O}\right) \mathrm{O}_{39}\right]^{5-}$ o heteroátomo primário é o $P$ (centro da estrutura) e o secundário é o Co (estando o ião Coll ligado a $\left[\mathrm{PW}_{11} \mathrm{O}_{39}\right]^{7}$ - e a uma molécula de $\mathrm{H}_{2} \mathrm{O}$ adicional).

Uma questão pertinente prende-se com o facto dos compostos baseados em polioxometalatos apresentarem, tipicamente, estruturas organizadas em unidades discretas. Pensa-se que a forte polarizaçăo dos átomos de oxigénios terminais em relação ao interior do heteropolianião (devido à ligação $\pi$ ), explica porque é que uma vez formado o heteropolianião, o processo de polimerização não se estende com a formação de cadeias (extensas), mas termina aí, com a obtenção de unidades discretas. [6] Por outro lado, podem-se considerar duas grandes sub-classes estruturais para os polioxometalatos: uma exibida pelos aniões de Mo (VI) e W (VI) e outra figura 3 Representação poliédrica da estrutura dos isómeros do anião de Keggin, $\beta$ e $\gamma$ ( $a$ e b, respectivamente) $e$ das espécies lacunares do anião de Keggin: monolacunar, $\alpha-\left[\mathrm{XM}_{11} \mathrm{O}_{39}\right](p+4)$ (c) e trilacunares, $\boldsymbol{\alpha}-\mathrm{A}-\left[\mathrm{XM}_{9} \mathrm{O}_{34}\right](\mathrm{p}+6)$ - (d)

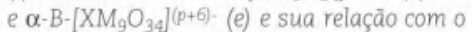
aniāo de Keggin (centro da figura). [2]

apresentada por certos polioxomolibdatos de valência mista (Mo (VI)/Mo (V)), conforme se verá no ponto 3 .

Os polioxometalatos apresentam um conjunto de propriedades peculiar. A sua dimensão iónica é relativamente grande tendo-se já isolado e caracterizado estruturalmente polioxometalatos até dimensões de $\sim 4 \mathrm{~nm}$ e as suas massas molares podem ir até $\sim 40000 \mathrm{~g} / \mathrm{mol}$. $[7,12]$ São robustos à degradação oxidativa e potenciais "armazenadores" de electrões, em virtude dos seus elementos constituintes fundamentais (Mo ou W) estarem, em geral, no seu estado de oxidação mais elevado. [2,4,6,11] Apresentam, geralmente, uma estabilidade térmica elevada. [4,13] A solubilidade dos sais de heteropolianiões é essencialmente determinada pelo contra-catião, uma vez que estes compostos possuem uma energia de rede baixa e a energia de solvatação do anião é igualmente baixa. [6] Assim, sais de $\mathrm{K}^{+}, \mathrm{Na}^{+}$, $\mathrm{NH}_{4}{ }^{+}$são solúveis em água; sais de tetra-n-butilamónio são solúveis nalguns solventes orgânicos polares, mas não em água. Sais com catiões surfactídeos são solúveis em solventes orgânicos apolares e os ácidos são geralmente solúveis em água e numa grande gama de solventes orgânicos. Esta característica 
é importante, designadamente, para a realização de experiências de catálise em fase líquida.

\section{O anião de Keggin e os seus derivados lacunares. Polioxomolibdatos de valência mista}

Entre os polioxometalatos mais estudados estão incluídos os aniões de Keggin. Este anião (que tomou o nome do autor que fez a sua caracterização estrutural) de fórmula geral $\alpha$ - $\left[\mathrm{XM}_{12} \mathrm{O}_{40}\right]^{\mathrm{p}-}\left(\mathrm{XM}_{12}\right)$, $M=M 0^{v i}, W^{v i} ; X=P^{v}, A^{v}, G^{l v}, S^{i v}, B^{\prime \prime \prime}$, Fell', Coll, $(\mathrm{H})_{2}{ }^{2+}$ (entre outros heteroátomos) apresenta uma simetria tetraédrica. Neste polioxometalato (figura 1c) o grupo central $\mathrm{XO}_{4}$ encontra-se rodeado por doze octaedros $\mathrm{MO}_{6}$, que formam quatro grupos de três octaedros cada $\left(\mathrm{M}_{3} \mathrm{O}_{13}\right)$. Observa-se a partilha de arestas de oxigénios (2) em cada grupo e de vertices de oxigénio (1) entre os vários grupos $\mathrm{M}_{3} \mathrm{O}_{13}$. [2,9] A partir do anião de Keggin (isómero $\alpha$ ) podem-se hipoteticamente derivar os isómeros $\beta, \gamma$ (figura $3 a, b), \delta$ e $\varepsilon$ obtidos por rotação de 60 . $^{\circ}$ de um grupo $\mathrm{M}_{3} \mathrm{O}_{13}$, relativamente ao isómero $\alpha$, sendo que, no caso do isómero $\varepsilon$, os quatro grupos $\mathrm{M}_{3} \mathrm{O}_{13}$ foram rodados. [2] Efectivamente, o isómero $\beta$ já foi observado (e isolado) para $\mathrm{PM}_{12} \mathrm{e}$ $\mathrm{SiM}_{12}, \mathrm{M}=\mathrm{Mo}, \mathrm{W}[2,14]$ e $\mathrm{o}$ anião $\gamma$ registado com $\mathrm{SiW}_{12}$ [15].

A partir do anião de Keggin (analogamente para os outros isómeros) podem-se ainda obter diversas estruturas lacunares por remoção de um ou mais octaedros. $\mathrm{O}$ anião $\alpha-\left[\mathrm{XM}_{11} \mathrm{O}_{39}\right]((\mathrm{p}+4)$ (monolacunar) resulta da remoção dum grupo $\mathrm{MO}^{4+}$ (um metal $\mathrm{M}$ conjuntamente com o seu oxigénio terminal), originando uma lacuna com 5 átomos de oxigénio potencialmente coordenantes (figura 3c). Os polianiōes $\alpha$ $\left[\mathrm{XM}_{9} \mathrm{O}_{34}\right]((\mathrm{p}+6)$ - (trilacunares) derivam da remoção de três octaedros (fragmento $\mathrm{M}_{3} \mathrm{O}_{6}{ }^{6+}$ ) do anião de Keggin (figura 3 d,e). Estas espécies químicas (monolacunares e trilacunares) são potencialmente coordenantes e originam com- plexos diversos, designadamente com catiões de metais de transição (entre outras espécies) (figura 1e). [2]

No caso dos polioxotungstatos a maior parte das estruturas conhecidas podem ser relacionadas com a do anião de Keggin (e seus isómeros) ou com fragmentos lacunares deste. Por exemplo, o anião de Dawson $\left[\mathrm{P}_{2} \mathrm{~W}_{18} \mathrm{O}_{62}\right]^{6-}$ (figura 1d), pode ser estruturalmente visualizado como o resultado da condensação de duas sub-unidades $\alpha-A-\left[\mathrm{PW}_{9} \mathrm{O}_{34}\right]^{9}$.

Para os polioxomolibdatos conhece-se uma maior diversidade estrutural, podendo estes exibir estruturas semelhantes às dos polioxotungstatos, assim como outras baseadas em unidades constituintes estruturalmente diferentes. É o caso dos aniões de valência mista, $\left\{\mathrm{Mo}_{154}\right\}$ e $\left\{\mathrm{Mo}_{176}\right\}$ (representação simplificada salientando apenas o numero de átomos de Mo da fórmula). [7,16,17] Estes polianiões de Mo (VI) e Mo (V) apresentam uma forma geométrica semelhante a um anel e são constituídos pelos fragmentos $\left\{\mathrm{Mo}_{8}\right\},\left\{\mathrm{Mo}_{2}\right\}$ e $\left\{\mathrm{Mo}_{1}\right\}$ (figura 2a). 0 fragmento $\left\{\mathrm{Mo}_{8}\right\}$ apresenta uma bipirâmide pentagonal central $\mathrm{MoO}_{7}$ ou $\mathrm{MoO}_{6} \mathrm{NO}$ à qual se encontram ligados cinco octaedros $\mathrm{MoO}_{6}$ por partilha de arestas de oxigénios, existindo mais dois octaedros adicionais, ligados por vértices de oxigénio; 0 fragmento $\left\{\mathrm{Mo}_{2}\right\}$ é constituido por dois octaedros partilhando um átomo de oxigénio, $\left\{\mathrm{MO}_{2} \mathrm{VIO}_{5}\left(\mathrm{H}_{2} \mathrm{O}\right)_{2}\right\}$ e o fragmento $\left\{\mathrm{Mo}_{1}\right\}$ corresponde a um octaedro $\mathrm{MoO}_{6}$. Nas estruturas resultantes, grupos $\left\{\mathrm{Mo}_{8}\right\}$ do mesmo plano do anel ligam-se entre si por intermédio dos fragmentos $\left\{\mathrm{Mo}_{2}\right\}$ e grupos $\left\{\mathrm{Mo}_{8}\right\}$ acima e abaixo do plano equatorial do anel estão ligados por intermédio dos fragmentos $\left\{\mathrm{Mo}_{1}\right\}$. De acordo com este esquema de construção geométrica os aniōes referidos podem ser representados como $\left.\left.\left[\left\{\mathrm{Mo}_{8}\right\} \mathrm{Mo}_{2}\right\} \mathrm{Mo}_{1}\right\}\right]_{\Pi}$, com $\mathrm{n}=14$ para $\left\{\mathrm{Mo}_{154}\right\}$, e $\mathrm{n}=16$ para $\left\{\mathrm{Mo}_{176}\right\}$. Outro anião estruturalmente relacionado é o $\left\{\mathrm{MO}_{248}\right\}$. [18] Neste aniāo a cavidade do anel de $\left\{\mathrm{Mo}_{176}\right\}$ aparece preenchida por dois fragmen- tos adicionais $\left\{\mathrm{MO}_{12} \mathrm{viMO}_{24} \mathrm{vO}_{96}\left(\mathrm{H}_{2} \mathrm{O}\right)_{24}\right\}$ $\equiv\left\{\mathrm{MO}_{36}\right\}$, ligados a oxigénios terminais dos grupos $\left\{\mathrm{MO}_{2}\right\}$.

Um outro polioxomolibdato de estrutura invulgar é 0 anião $\left\{\mathrm{Mo}_{132}\right\}$ (figura $2 \mathrm{~b}$ ). [8] Este aniăo baseia-se no fragmento fundamental $\left\{\left(\mathrm{Mov}^{\mathrm{v}}\right) \mathrm{Mo}_{5} \mathrm{vi}_{21}\left(\mathrm{H}_{2} \mathrm{O}\right)_{6}\right\} \equiv$ $\left\{\mathrm{Mo}_{6}\right\}$, que corresponde a uma bipirâmide pentagonal central $\mathrm{MoO}_{7}$ ligada a cinco octaedros $\mathrm{MoO}_{6}$ por partilha de arestas, formando um "pentágono". Os vários fragmentos $\left\{\mathrm{Mo}_{6}\right\}$ ligam-se entre si por intermédio de pontes $\left\{\mathrm{Mo}_{2} \mathrm{O}_{4}\left(\mathrm{CH}_{3} \mathrm{COO}\right)\right\}$, originando um anião de forma aproximadamente esférica. A estrutura deste anião tem semeIhança geométrica com a do fulereno $\mathrm{C}_{60}$, apresentando, contudo, uma dimensão consideravelmente superior (diâmetro de cerca de $2.5 \mathrm{~nm}$ - nanocápsula).

Todos estes polioxomolibdatos referidos, $\left\{\mathrm{Mo}_{154}\right\},\left\{\mathrm{Mo}_{176}\right\},\left\{\mathrm{Mo}_{248}\right\}$ e $\left\{\mathrm{Mo}_{132}\right\}$ apresentam dimensões nanométricas e massas molares superiores a 20000 $\mathrm{g} / \mathrm{mol}$ e estão (juntamente com outros polioxometalatos) entre as espécies inorgânicas discretas de maior tamanho, que se encontram estruturalmente caracterizadas.

\section{Propriedades electrónicas}

Os polioxometalatos são susceptiveis de receber electrões, em processos reversíveis ou irreversiveis. Os polioxometalatos baseados em octaedros de coordenação $\mathrm{M}^{\mathrm{V}} \mathrm{O}_{6}$ com apenas uma ligação terminal $\mathrm{M}=\mathrm{O}$ (tipo I), onde estão incluídos, por exemplo, os aniōes de Keggin, apresentam em solução aquosa, voltamogramas cíclicos caracterizados por várias ondas de redução reversíveis de um ou dois electrões cada. A sua redução origina espécies de valência mista, tipicamente de cor azul escura (até um máximo de seis electrões adicionados). Em solução aquosa, observa-se a estabilização de espécies reduzidas por protonação e conhecem-se fenómenos de dismutação (equaçăo I). [2,4] Estudos electroquímicos realizados em solventes 
orgânicos para aniões do tipo Keggin evidenciam alterações significativas dos potenciais de redução, quando comparados com os correspondentes em solução aquosa. $[19,20]$ Em contraste, polioxometalatos baseados em octaedros de coordenação $\mathrm{M}^{\mathrm{v}} \mathrm{O}_{6}$ com duas ligações terminais cis $\mathrm{M}=0$ (tipo II) (figura 1b) sāo reduzidos de uma forma irreversível ou são electroquímicamente inactivos. [2,4]

As espécies reduzidas de polioxometalatos do tipo I, vulgarmente conhecidas por "heteropolianiões azuis", apresentam um espectro óptico caracterizado pela presença de bandas de transferência de carga metal-metal, $\left(\mathrm{M}^{\vee} \rightarrow \mathrm{M}^{\mathrm{v}}\right)$, de absortividade molar elevada, na região do Vis/IV próximo. Realce-se que esta característica é a base para o uso de vários polioxometalatos em determinações analíticas por espectrofotometria (Si, P, Ge, As). Por outro lado, o Mov, W (ou o Viv) apresentam sinais caracteristicos no espectro de Ressonância Paramagnética Electrónica (RPE) podendo ser a sua presença estudada por essa técnica. Em geral, à temperatura ambiente existe uma rápida deslocalização electrónica (mais rápida que a escala de tempo do RPE) nas espécies reduzidas de um electrão, como consequência da transferência electrónica intra-molecular, em que o número e o tipo de centros metálicos envolvidos na troca depende da estrutura e composição do complexo; a temperatura suficientemente baixa (77 K ou inferior), o electrão desemparelhado fica "localizado" num dado centro metálico. A redução de dois ou quatro electrões origina espécies silenciosas no RPE, devido ao emparelhamento de spin electrónico. $[2,4,6]$

\section{Síntese}

Os polioxometalatos são preparados geralmente com base em reacções realizadas em solução aquosa ou em solvente orgânico (ou em misturas de solventes). Grande parte das sínteses efectuadas em solução aquosa baseia-se numa acidificação controlada do meio reaccional (equações II e III). Espécies lacunares dos heteropolianiões de Keggin e Dawson, podem ser obtidas por adição de soluções alcalinas a soluções aquosas contendo esses aniões (equação IV). [2,21] A presença de agentes redutores é favorável à obtenção de espécies reduzidas e/ou novas estruturas de polioxometalatos. [11,12]

Por outro lado, a adição de solvente orgânico, pode permitir a preparação de polioxometalatos pouco estáveis em solução aquosa. Por exemplo, o anião $\beta-\left[\mathrm{PW}_{12} \mathrm{O}_{40}\right]^{3}$ - foi obtido com base numa reacção feita numa mistura de solventes água/acetonitrilo (a partir de tungstato, hidrogenofosfato e ácido clorídrico),verificando-se em solução aquosa que o correspondente isómero $\beta$ se trans-forma espontaneamente no isómero $\alpha$. [14] Analogamente, o anião $\gamma-\left[\mathrm{SiW}_{12} \mathrm{O}_{40}\right]^{4-}$ foi preparado a partir de uma reacção realizada numa mistura água/etanol. [15]

$\mathrm{O}$ isolamento dos polioxometalatos é feito, em geral, através da precipitação com sais de catiões de metais alcalinos $\left(\mathrm{Li}^{+}, \mathrm{K}^{+}, \mathrm{Na}^{+}, \mathrm{Cs}^{+}\right)$ou sais de alquilamónio (ex: tetra- $n$-butilamónio). 0 uso de contra-catiões adequados ou combinações de diferentes contra-catiōes pode ser determinante no isolamento de um determinado tipo de polioxometalato e na obtenção de cristais adequados para caracterização estrutural por difracção de raios $X$. É de realçar, ainda, que as sinteses de compostos com polioxometalatos podem ser dimensionadas para a obtenção de grandes quantidades de composto, utilizando reagentes comuns e relativamente baratos, validando assim o interesse para aplicaçōes do foro industrial.

\section{Aplicações em catálise. Outras aplicações}

Uma das áreas de investigação onde os polioxometalatos apresentam mais potencial é na catálise, podendo ser utilizados quer como catalisadores ácidos ou oxidativos. A catálise ácida remonta aos anos 70 , quando se descobriu que certos heteropoliácidos baseados no anião de Keggin, $\mathrm{H}_{n}\left[\mathrm{XM}_{12} \mathrm{O}_{40}\right](X=P, \mathrm{Si}$, $M=M o, W$ ) eram ácidos de Brönsted mais fortes que o ácido sulfúrico, tendo a vantagem de não serem voláteis e de possuirem uma boa estabilidade térmica. Desde então, foram utilizados num grande número de transformações químicas, sendo de destacar o seu uso actual na indústria nos processos de hidratação do propeno (formação de álcool) e polimerização do tetra-hidrofurano. [22-24]

Os polioxometalatos revelam-se, igualmente, catalisadores eficientes em numerosas transformações oxidativas. Uma lista não exaustiva de oxidações com oxigénio molecular, usando os molibdovanadatos com a estrutura de Keggin, $\left[\mathrm{PMO}_{12-x} \mathrm{~V}_{\mathrm{x}} \mathrm{O}_{40}\right](\mathrm{x}=1-6)$, inclui a oxidação de 2-metilnaftaleno a 2-metil1,4-naftoquinona (vitamina $K_{3}$, esquema 1-A), oxidaçăo de 2,3,5-trimetilfenol a 2,3,5-trimetil-1,4-benzoquinona (precursor da vitamina E), oxidação de compostos de enxofre (poluentes), oxidação de acetileno a acetaldeído (oxidação de Wacker, um processo industrial), oxidação da metacroleína a ácido metacrílico (processo industrial) e deslenhificaçăo da pasta kraft (branqueamento da pasta celulósica). As oxidações com $\mathrm{O}_{2}$ podem ser realizadas com os polioxometalatos dissolvidos em fase líquida ou imobilizados em matrizes de carvão activado, sílica, ou outros suportes (sistema heterogéneo). [22,25]

Os polioxometalatos têm sido, ainda, empregues como catalisadores oxidativos usando como oxidante o peróxido

$$
\begin{aligned}
& 8 \mathrm{H}^{+}+7 \mathrm{WO}_{4}^{2-} \rightarrow\left[\mathrm{W}_{7} \mathrm{O}_{24}\right]^{6-}+4 \mathrm{H}_{2} \mathrm{O} \\
& 12 \mathrm{MoO}_{4}^{2-}+\mathrm{HPO}_{4}^{2-}+23 \mathrm{H}^{+} \rightarrow\left[\mathrm{PMO}_{12} \mathrm{O}_{40}\right]^{3-}+12 \mathrm{H}_{2} \mathrm{O} \\
& {\left[\mathrm{P}_{2} \mathrm{~W}_{18} \mathrm{O}_{62}\right]^{6-}+6 \mathrm{OH}^{-} \rightarrow\left[\mathrm{P}_{2} \mathrm{~W}_{17} \mathrm{O}_{61}\right]^{10-}+\mathrm{WO}_{4}^{2-}+3 \mathrm{H}_{2} \mathrm{O}}
\end{aligned}
$$

equação II

equação III

equação IV 
esquema 1 Algumas oxidações de compostos orgânicos catalisadas por polioxometalatos. $[22,27,28]$
A<smiles>Cc1ccc2ccccc2c1</smiles><smiles>CC1=CC(=O)c2ccccc2C1=O</smiles>

B

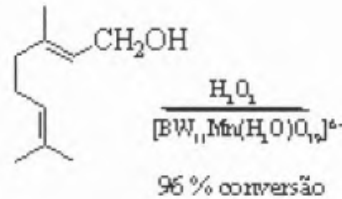

$\mathrm{C}$<smiles>C1CCC2CCCC2C1</smiles>

$\frac{\mathrm{H}_{2} \mathrm{O}_{2}}{\left[\mathrm{PWT}_{11} \mathrm{Re}\left(\mathrm{H}_{2} \mathrm{O}\right) \mathrm{O}_{12}\right]}$

$76 \%$ comersão

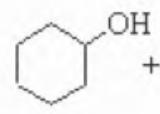

$6 \%$<smiles>CC(C)=CCCC(C)(C)CO</smiles><smiles>O=C1CCCCC1C1CCCCC1O</smiles>

$57 \%$ de hidrogénio, que a seguir ao oxigénio é o mais viável (barato e "amigo" do ambiente). Epoxidação de alcenos como o ciclo-octeno e ciclo-hexeno, de terpenos como o geraniol (esquema 1-B) ou oxidação de ciclo-alcanos (esquema 1-C) foram realizadas com sucesso, usando polioxometalatos substituídos por metais de transição. [25,26-28]. As reacções com $\mathrm{H}_{2} \mathrm{O}_{2}$ são normalmente realizadas em sistema monofásico (fase líquida) ou bifásico (duas fases líquidas imiscíveis). Alternativas a este procedimento incluem a imobilização dos polioxometalatos em suportes de silica funcionalizada, o que apresenta vantagens quer pela possibilidade de recuperar facilmente 0 catalisador no final da reacção quer pela ausência desejada do solvente orgânico. [29] 0 uso de sistemas heterogéneos poderá ser um meio de expandir a aplicação dos polioxometalatos a uma escala mais alargada, nas reacções catalíticas com peróxido de hidrogénio. É de frisar que as múltiplas possibilidades que existem de fazer variar a estrutura do polioxometalato e em particular para um dado tipo de estrutura, a sua composição, estão, ainda, por ser exploradas no campo da catálise oxidativa.

Os polioxometalatos têm apresentado algum interesse no campo da medicina, designadamente como agentes anti-virais. Um grande número de resultados pode-se encontrar na literatura, regis- tando a sua acção contra o HIV (vírus da imunodeficiência adquirida), RSV (vírus respiratório sincicial), vários tipos de vírus relacionados com a leucemia, herpes, entre outros, existindo evidência da sua acção não apenas in vitro mas também in vivo. Demonstrou-se ainda que os polioxometalatos são capazes de penetrar nas membranas celulares e localizar-se no interior das células, sendo a sua actividade anti-viral relacionada com a interacção que apresentam com enzimas virais ou proteínas de superfície virais (ex: glicoproteína 120 do HIV). Factores como a estrutura, composição química, carga iónica e tamanho do polioxometalato, para além das características do vírus são de considerar na sua acção anti-viral. [5,30]

Outras aplicações dos polioxometalatos, para além das já referidas na catálise e medicina, incluem o seu uso em química analítica (determinação de P, Si, As, proteínas), em revestimentos de superfícies metálicas resistentes à corrosão, para tratamento de soluções radioactivas, na preparação de eléctrodos selectivos, sensores de gás (ex: $\mathrm{CO}$ ) ou células de combustível, utilização em pigmentos para tintas ou na obtenção de materiais com propriedades ópticas (fotocromismo, electrocromismo, óptica não linear). A título de curiosidade refira-se que o número de patentes relacionadas com polioxometalatos entre 1991 e 1996 foi sempre superior a 100 por ano, o que é, de alguma forma, ilustrativo das potencialidades deste tipo de compostos. [31-33]

\section{Conclusões}

Os polioxometalatos formam uma classe de aniões que apresenta um conjunto de propriedades topológicas e electrónicas invulgar. Em particular, certos polioxomolibdatos recentemente obtidos, como o $\left\{\mathrm{Mo}_{132}\right\}$, dada a sua estabilidade em solução e propriedades estruturais, apresentam um interesse acrescido pela possibilidade de neles se desenvolverem interacções hóspede-hospedeiro (nanocápsula) um assunto de interesse potencial, ainda num estado embrionário. [34]

A utilização em áreas de impacto social como a medicina ou a ciência dos materiais abre portas para uma investigação continuada e de preferência interdisciplinar, que deve ser desenvolvida no futuro. À parte isso, a beleza estética de certas arquitecturas moleculares revela-se singular; citando uma frase de Muller, a propósito da descoberta do $\left\{\mathrm{Mo}_{132}\right\}$ :

"Of interest is certainly not only the production of mesoscopic (or nanostructured) and macroscopic objects with func- 
tional versatility, but also the aesthetic beauty of such assemblies" [8]

\section{Agradecimentos}

Agradece-se a cortesia dos Professores Achim Muller e Michael Pope.

\section{Referências}

1. Evans, H. T. Perspect. Struct. Chem., $1971,4,1$.

2. Pope, M. T. Heteropoly and Isopoly Oxometalates, Springer Verlag, 1983.

3. Pope, M. T. no Comprehensive Coordination Chemistry, Wilkinson, G. (ed.) Pergamon Press, 1987, vol. 3, p. 1023.

4. Pope, M. T.; Muller, A. Angew. Chem., Int. Ed. Engl., 1991, 30, 34.

5. Polyoxometalates: From Platonic Solids to Anti-rectroviral Activity, Pope, M. T.; Muller, A. (eds.), Kluwer Academic Publ., 1994.

6. Baker, L. C. W.; Glick, D. C. Chem. Rev., 1998, 98, 3.

7. Muller, A.; Krickemeyer, E; Bogge, H.; Schmidtmann, M.; Beugholt, C.; Kogerler, P.; Lu, C. Angew. Chem., Int. Ed. Engl., 1998, 37, 1220.

8. Muller, A.; Krickemeyer, E; Bogge, H.; Schmidtmann, M.; Peters, F. Angew. Chem., Int. Ed. Engl., 1998, 37, 3360.
9. Keggin, J. F. Nature, 1933, 131, 908. 10. Hill, C. L. Chem. Rev., 1998, 98, 1.

11. Muller, A. em NATO ASI on Polyoxometalate Molecular Science, Tenerife, 2001.

12. Muller, A.; Peters, F.; Pope, M. T.; Gatteschi, D. Chem. Rev., 1998, 98, 239.

13. Gamelas, J. A. F.; Couto, F. A.; Trovāo, M. C.; Cavaleiro, A. M. V.; Cavaleiro, J. A. S.; Pedrosa de Jesus, J. D. Thermochim. Acta, 1999, 326, 165

14. Himeno, S.; Takamoto, M.; Ueda, T. J. Electroanal. Chem., 1999, 465, 129.

15. Tézé, A.; Canny, J.; Gurban, L.; Thouvenot, R.; Hervé, G. Inorg. Chem., 1996, 35, 1001.

16. Muller, A.; Krickemeyer, E; Meyer, J.; Bogge, H.; Peters, F.; Plass, W.; Diemann, E.; Dillinger, S.; Nonnenbruch, F. Randerath, M.; Menke, C. Angew. Chem., Int. Ed. Engl., $1995,34,2122$

17. Muller, A.; Beugholt, C. Nature, 1996, $383,296$.

18. Muller, A.; Shah, S. Q.; Bogge, H.; Schmidtmann, M. Nature, 1999, 397, 48.

19. Balula, M. S.; Gamelas, J. A. F.; Carapuça, H. M.; Cavaleiro, A. M. V.; Schlindwein, W. aceite para publicação em Eur. J. Inorg chem.

20. Gamelas, J. A. F.; Balula, M. S.; Carapuça, H. M.; Cavaleiro, A. M. V. Electrochem. Commun., 2003, 5, 378.

21. Klemperer, W. Inorg. Synth., 1990, 27, 71.

22. Kozhevnikov, I. Chem. Rev., 1998, 98, 171.
23. Mizuno, N.: Misono, M. Chem. Rev. $1998,98,199$

24. Misono, M.; J. Chem. Soc., Chem. Commun., 2001, 1141.

25. Neumann, R. Prog. Inorg. Chem., 1998 $47,317$.

26. Hill, C. L.; Zhang, X. Nature, 1995, 373, 324.

27. Simões, M. M. Q.; Conceição, C. M. M.; Gamelas, J. A. F.; Domingues, P. M. D.; Cavaleiro, A. M. V.; Cavaleiro, J. A. S.; FerrerCorreia, A. J. V.; Johnstone, R. A. W. J. Mol. Catal. A, 1999, 144, 461

28. Santos, I. C. M.; Simões M. M. Q.; Pereira, M. M. M; Martins, R. R. L.; Neves, M. G. P.; Cavaleiro, J. A. S.; Cavaleiro, A. M. V. J. Mol. Catal. A, 2003, 195, 253.

29. Neumann, R.; Miller, H. J. Chem. Soc., Chem. Commun., 1995, 2277.

30. Rhule, J. T.; Hill, C. L.; Judd, D; Schinazi, R. Chem. Rev. 1998, 98, 327.

31. Katsoulis, D. E. Chem. Rev. 1998, 98, 359.

32. Coronado, E.; Gómez-Garcia, C. Chem. Rev. 1998, 98, 273.

33. Yamase, T. Chem. Rev. 1998, 98, 307. 34. Muller, A.; Samar, K; Kogerler, P.; Bogge, H.; Schmidtmann, M.; Trautwein, A.; Shunemann, V.; Krickemeyer, E; Preetz, W. Angew. Chem., Int. Ed. Engl., 2000, 39, 3414. 


\section{Eur \\ European Journal of Inorganic Chemistry}

\section{Yourjournal}

First Impact Factor: 2.222

Rapid publication times especially for short communications

Now supported by ten national chemical societies (Belgium, France, Germany, Greece, Hungary - together with the Hungarian Academy of Sciences, Italy, The Netherlands, Portugal, Spain) Authors' work exhibited on the cover Attractive personal member subscription rates available; see: www.EurJIC.com

More color

\section{European Journal of Inorganic Chemistry}

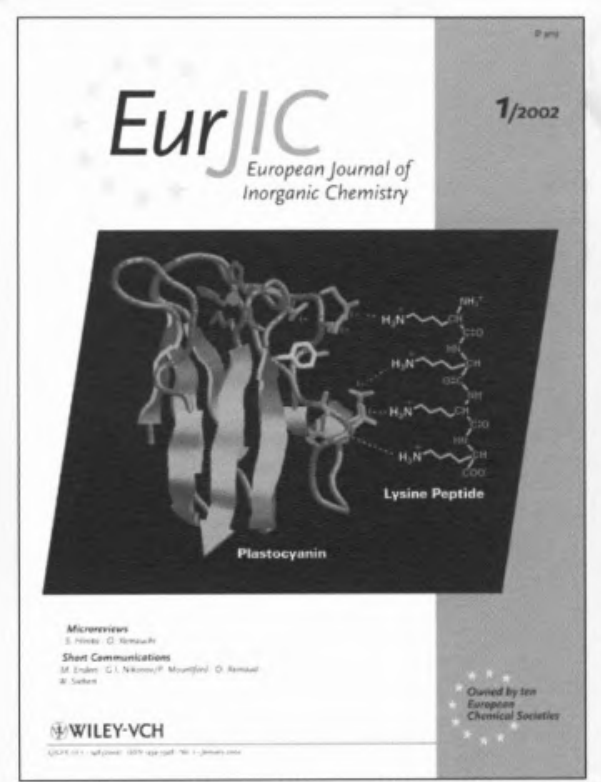

Wiley-VCH

200212 issues

ISSN Print 1434-1948

ISSN Electronic 1099-0682

The European Journal of Inorganic

Chemistry publishes full papers and

short communications on the

entire spectrum of inorganic and

organometallic chemistry. These

contributions are supplemented by

microreviews - introducing readers

to one specific area of an author's

research by means of a detailed

overview of one selected topic.

Senior Editor: Ivano Bertini (Italy)

FREE SAMPLE COPY

The European Journal of Inorganic Chemistry is available online through Wiley InterScience. Visit Wiley InterScience (www.interscience.wiley.com) for complete details and see the FREE full text virtual sample copy.
Order Your Copy now:

Just copy, fill in and fax to: $+49 /(0) 6201 / 606-172$

J Please send me a free sample print copy

」 Please enter our/my 2002 subscription to:

European Journal of Inorganic Chemistry

2002, ISSN Print 1434-1948

2002, ISSN Electronic 1099-0682

at the institutional rate*:

print electronic

」 Europe $\lrcorner € 1868 \quad J € 1868$

J Switzerland J sFr 3258 J sFr 3258

\lrcorner All other

countries JUS\$2308 JUS\$2308

* For a $5 \%$ premium, institutions can choose both print and electronic access. $]$

For members of the owner societies from Belgium, France, Germany, Greece, Hungary, Italy, The Netherlands, Portugal and Spain, at the personal rate:

Europe print
\lrcorner$\in 218$
Switzerland $\lrcorner \mathrm{sFr} 458$
\lrcorner All other
countries $\lrcorner$ US\$ 248

Prices include postage and handling charges.

Please tick: $\square$ private $\square$ business

Name

Address

City/Postcode

Country

Membership-No.

Date/Signature

Please return this order to your local bookseller or to:

Customers in Germany, Austria and Switzerland:

Wiley-VCH Customer Service

P.O. Box 101161 ,

D-69451 Weinheim, Germany Phone: +49 (0) 6201-606147

Fax: +49 (0) 6201-606172 e-mail: subservice@wiley-vch.de

Customers in all other areas: John Wiley \& Sons, Ltd. Journals Administration Department 1 Oldlands Way

Bognor Regis West Sussex, P022 9SA, England

Phone: +44 (0) 1243-779 777

Fax: $+44(0) 1243-843232$

e-mail:cs-journals@wiley.co.uk 